\title{
ON A PAPER OF K. SOUNDARARAJAN ON SMOOTH NUMBERS IN ARITHMETIC PROGRESSIONS
}

\author{
ADAM J HARPER
}

\begin{abstract}
In a recent paper, K. Soundararajan showed, roughly speaking, that the integers smaller than $x$ whose prime factors are less than $y$ are asymptotically equidistributed in arithmetic progressions to modulus $q$, provided that $y^{4 \sqrt{e}-\delta} \geq q$ and that $y$ is neither too large nor too small compared with $x$. We show that these latter restrictions on $y$ are unnecessary, thereby proving a conjecture of Soundararajan. Our argument uses a simple majorant principle for trigonometric sums to handle a saddle point that is close to 1 .
\end{abstract}

\section{INTRODUCTION}

For $y \geq 1$, let $\mathcal{S}(y)$ denote the set of $y$-smooth numbers: that is, the set of numbers all of whose prime factors are less than or equal to $y$. For $x \geq 1$, and natural numbers $a, q$, we define the following counting functions:

$$
\Psi_{q}(x, y):=\sum_{n \leq x,(n, q)=1} \mathbf{1}_{\{n \in \mathcal{S}(y)\}}, \quad \text { and } \quad \Psi(x, y ; q, a):=\sum_{n \leq x, n \equiv a(\bmod q)} \mathbf{1}_{\{n \in \mathcal{S}(y)\}},
$$

where 1 denotes the indicator function.

In his 2008 article [8], K. Soundararajan makes the following equidistribution conjecture:

Conjecture 1 (Soundararajan, 2008). Let $A$ be a given positive real number. Let $y$ and $q$ be large with $q \leq y^{A}$, and let $(a, q)=1$. Then as $\log x / \log q \rightarrow \infty$ we have

$$
\Psi(x, y ; q, a) \sim \frac{1}{\phi(q)} \Psi_{q}(x, y) .
$$

As Soundararajan [8] discusses, in our current state of knowledge about character sums it would be very hard to prove the conjecture for $A \geq 4 \sqrt{e}$; for if the conjecture is true, then e.g. the least quadratic non-residue modulo $q$ must lie below $q^{1 / A}$. However, Soundararajan is able to prove the conjecture for $A<4 \sqrt{e}$, on the additional assumption that $e^{y^{1-\epsilon}} \geq x \geq y^{(\log \log y)^{4}}$. In this note we establish the following result, confirming that this assumption on $y$ is not needed.

Date: 10 th March 2011.

The author is supported by a studentship from the Engineering and Physical Sciences Research Council of the United Kingdom. 
Theorem 1. Let $\delta>0$, and suppose that $y \leq x$, that $2 \leq q \leq y^{4 \sqrt{e}-\delta}$, and $(a, q)=1$. If $y$ is large enough depending on $\delta$, then as $\log x / \log q \rightarrow \infty$ we have

$$
\Psi(x, y ; q, a) \sim \frac{1}{\phi(q)} \Psi_{q}(x, y) .
$$

In fact, following Soundararajan [8], we work with a smooth weight function $\Phi(n / x)$ throughout: see $\S 2.1$ for further details. We obtain a smoothly weighted version of Theorem 1 with a quantitative error term

$$
O_{\Phi}\left(\frac{\Psi_{q}(x, y)}{\phi(q)}\left(\min \left\{\frac{q \sqrt{y}}{(\log \log x)^{1 / 3}}, \frac{1}{\log y}\right\}+\frac{\log q}{u^{c} \log y}+\frac{\log w}{\left(c \delta w^{\delta / 2}\right)^{c \log (2+(\log x) / y)}}\right)\right),
$$

where $c>0$ is an absolute constant, and we write $u=\log x / \log y, v=\log x / \log q$ and $w=\min \{v, y\}$. We caution that the reader should not try to read off the necessary dependence of $y$ on $\delta$ from this bound, as it is not valid unless $y$ is large enough that the error term $O_{\delta}\left(y^{-\delta^{2}} \log ^{2} y\right)$ in Character Sum Bound 1 (see $\S 2.5$, below) is smaller than $\delta / 2$, say.

Soundararajan's article [8] also contains an 'equidistribution in cosets' result, which gives information towards Conjecture 1 in the case $A \geq 4 \sqrt{e}$. This is again proved on the assumption that $e^{y^{1-\epsilon}} \geq x \geq y^{(\log \log y)^{4}}$, which we can now remove.

Theorem 2. Let $A$ be a given positive real number, and let $y$ and $q$ be large with $q \leq y^{A}$. There is a subgroup $H$ of $(\mathbb{Z} / q \mathbb{Z})^{*}$, of index bounded in terms of $A$ only, such that whenever $a, b \in(\mathbb{Z} / q \mathbb{Z})^{*}$ satisfy $a / b \in H$ we have

$$
\Psi(x, y ; q, a)-\Psi(x, y ; q, b)=o\left(\Psi_{q}(x, y) / \phi(q)\right) \quad \text { as } \log x / \log q \rightarrow \infty .
$$

We will not say much about Theorem 2, which follows from the proof of Theorem 1 as in Soundararajan's paper [8]. The author has tried to keep explicit dependence on $A$ in the arguments below, so a keen reader may check that provided $\log y /((A+1) \log (A+2))$ and $\log v /((A+1) \log (A+2))$ are sufficiently large we have a smoothly weighted version with a better error term than above (the term involving $w$ can be removed). The error term is better in Theorem 2 because we do not need Character Sum Bound 1 for the proof. In our first appendix we comment briefly on how to pass from the smoothly weighted to the unweighted version of Theorem 2 , which seems to require a slightly different procedure 1 now that $y$ is unrestricted in terms of $x$.

Soundararajan [8] argues, roughly, by observing the usual decomposition

$$
\Psi(x, y ; q, a)=\frac{1}{\phi(q)} \sum_{\chi(\bmod q)} \overline{\chi(a)} \Psi(x, y ; \chi), \quad \text { where } \quad \Psi(x, y ; \chi):=\sum_{n \leq x} \chi(n) \mathbf{1}_{\{n \in \mathcal{S}(y)\}},
$$

\footnotetext{
${ }^{1} \mathrm{~A}$ reader who is checking Theorem 2 may wish to consult this appendix first. The unsmoothing procedure that we use will allow one to prove Theorem 2 without needing to analyse the characters $\chi \in \mathcal{B}$, (defined in $\S 2.5$, below), which is quite a helpful simplification.
} 
and analysing $\Psi(x, y ; \chi)$ using knowledge of the $L$-series $L(s, \chi)$. His key innovation, perhaps, is to exploit the fact that we are interested in all characters to modulus $q$ taken together, and that we can make much stronger statistical statements than we can statements about individual $L$-series.

Perhaps surprisingly, it is when $y$ is close to $x$ that Soundararajan's argument is difficult to extend. This is because of a 'saddle point problem': as $y$ approaches $x$, the (Euler product) terms from which one can gain by making non-trivial estimations carry progressively less weight, so it is important not to lose anywhere else. To achieve this we avoid applying absolute value bounds to integrals, and instead exploit a majorant principle for trigonometric sums. See $\S \S 2.3-2.5$. It is the author's opinion that this argument is the most interesting new aspect of this work.

A further difficulty in establishing Theorem 1 is that two parts of Soundararajan's proof, his "basic argument" and "Rodosskil argument", are valid respectively when a quantity $k$ (explained in $\S 2.1$ ) is quite large depending on $x$, or is quite small depending on $u$. When $y$ approaches $x$ a gap emerges between these ranges, and to deal with this we need an argument based on Taylor expansion and a smoothed explicit formula. See $\S 2.3$ and $\S 3$. When $y$ is small Soundararajan's proofs [8] almost go through, except for minor technical problems and some difficulties if $y$ does not tend to infinity with $x$. In $\S \S 2.6-2.7$ we give an argument that addresses these problems.

The author has tried to write this note in a reasonably self-contained way, whilst not simply repeating arguments that appear in Soundararajan's paper [8]. To this end, three important pieces of ' $L$-function information' obtained by Soundararajan are stated without proof in $\S 2$, as Rodosskiu Bound 1, Rodosskiı̌ Bound 2 and Character Sum Bound 1. Except in the application of these bounds, (and the general set-up, which we recall in §2.1), many details of our argument are different from that of Soundararajan [8], and so we give a detailed account.

Since it adds very little complication, and may be illuminating, we shall prove Theorem 1 for all $y$, and not only the range not covered by Soundararajan's results. We distinguish in our work between "large $y$ ", namely $e^{\log ^{1 / 10} x}<y \leq x$; "small $y$ ", namely $(\log \log x)^{3} \leq y \leq e^{\log ^{1 / 10} x}$; and "very small $y$ ", namely $y<(\log \log x)^{3}$.

If $q<\sqrt{y}$, say, a result of Granville [3] shows $\Psi(x, y ; q, a)$ is $\Psi_{q}(x, y) \phi(q)^{-1}(1+$ $\left.O\left(\log ^{-1} y\left(1+u^{-c} \log q\right)\right)\right)$. We invoke this result except when $q \sqrt{y} \leq(\log \log x)^{1 / 3}$ (for which see $\S 2.7$ ), and can therefore always assume that $q \geq \sqrt{y}$ except in that case. This will be convenient in applying various $L$-function computations, so that $\log q$ is somewhat comparable to $\log y$. The reader should also bear in mind, when checking that our proof supplies the bound claimed, that if $\sqrt{y} \leq q \leq y^{4 \sqrt{e}}$ then $u=\log x / \log y$ is comparable in size to $v=\log x / \log q$. 


\section{Overview OF THE ARGUMENT}

2.1. Initial set-up. This subsection records some preliminary observations, mostly from $\S 2$ of Soundararajan's paper [8] (which may be consulted for a more detailed description).

Let $\Phi:[0, \infty) \rightarrow[0,1]$ be a function supported on $[0,2]$, which equals 1 on $[0,1 / 2]$, and which is nine times continuously differentiable (say). We set

$$
\Psi(x, y ; q, a, \Phi):=\sum_{n \in \mathcal{S}(y), n \equiv a(\bmod q)} \Phi(n / x),
$$

which has a decomposition into weighted character sums $\Psi(x, y ; \chi, \Phi)$ as in the introduction. We will work to show that $\Psi(x, y ; q, a, \Phi)$ is approximately equal for all $(a, q)=1$, and by choosing $\Phi$ to bound $\mathbf{1}_{[0,1]}$ from above and then below (in a way explained further in our first appendix) this will imply Theorem 1.

We define a truncated Euler product corresponding to $y$-smooth numbers, viz.

$$
L(s, \chi ; y):=\prod_{p \leq y}\left(1-\frac{\chi(p)}{p^{s}}\right)^{-1}=\sum_{n \in \mathcal{S}(y)} \frac{\chi(n)}{n^{s}}, \quad \Re(s)>0 .
$$

Then, as usual, we can represent $\Psi(x, y ; \chi, \Phi)$ as a contour integral involving $L(s, \chi ; y)$ :

$$
\Psi(x, y ; \chi, \Phi)=\frac{1}{2 \pi i} \int_{c-i \infty}^{c+i \infty} L(s, \chi ; y) x^{s} \breve{\Phi}(s) d s, \quad c>0,
$$

where $\breve{\Phi}(s)=\int_{0}^{\infty} \Phi(t) t^{s-1} d t$ is the Mellin transform of $\Phi$. Because $\Phi$ is so smooth, integration by parts shows $|\breve{\Phi}(s)| \ll_{\Phi}|s|^{-1}(|s|+1)^{-8}$ for $\Re(s)>0$, which will be used several times. We also note that $\breve{\Phi}(c) \geq \int_{0}^{1 / 2} t^{c-1} d t \geq 1 /(2 c)$ for $0<c \leq 1$.

We choose $c$ to be $\alpha=\alpha(x, y)$, a quantity coming from a saddle-point argument of Hildebrand and Tenenbaum [5] (that was extended2 to treat $\Psi_{q}(x, y)$ by de la Bretèche and Tenenbaum [2]). In practice this means the following: provided that $y, u=$ $\log x / \log y$ and $\log ^{2} y / \log q$ (say) are larger than certain absolute constants, as we assume throughout, we have

$$
\alpha(x, y)=\left\{\begin{array}{ll}
1-\frac{\log (u \log u)}{\log y}+O\left(\frac{1}{\log y}\right) & \text { if } y>\log x \\
\Theta\left(\frac{y}{u \log ^{2} y}\right) & \text { otherwise }
\end{array},\right.
$$

and, writing $\chi_{0}$ for the principal Dirichlet character to modulus $q$, we have

$$
\Psi\left(x, y ; \chi_{0}, \Phi\right) \gg \frac{x^{\alpha} L\left(\alpha, \chi_{0} ; y\right) \breve{\Phi}(\alpha)}{\sqrt{2 \pi(1+\log x / y) \log x \log y}} .
$$

\footnotetext{
${ }^{2}$ Hildebrand and Tenenbaum [5] studied $\Psi(x, y):=\sum_{n \leq x} \mathbf{1}_{\{n \in \mathcal{S}(y)\}}$, and later de la Bretèche and Tenenbaum [2] showed that some 'obvious' adaptations of their results also hold for $\Psi_{q}(x, y)$, on a wide range of $q$. The bound that we record for $\Psi\left(x, y ; \chi_{0}, \Phi\right)$, involving the smoothing $\mathbf{1}_{[0,1 / 2]} \leq \Phi \leq \mathbf{1}_{[0,2]}$, is an easy consequence of Théorème 2.1 of de la Bretèche and Tenenbaum [2] (e.g. because, as in our first appendix, $\Psi_{q}(x / 2, y)$ is comparable in size to $\left.\Psi_{q}(2 x, y)\right)$.
} 
Finally we present some notation concerning the zeros of the $L$-series $L(s, \chi)$. For $0 \leq k \leq(\log q) / 2$, write

$$
\begin{gathered}
\Xi(k):=\left\{\chi: \chi \neq \chi_{0}, L(\sigma+i t, \chi) \neq 0 \text { for } \sigma>1-\frac{k}{\log q},|t| \leq q \text {, but } L(\sigma+i t, \chi)=0\right. \\
\text { for some } \left.\sigma>1-\frac{k+1}{\log q},|t| \leq q\right\} .
\end{gathered}
$$

As Soundararajan [8] describes, the so-called log-free zero density estimate implies that $\# \Xi(k) \leq C_{1} e^{C_{2} k}$ for all $k$, for certain absolute constants $C_{1}, C_{2}$. Thus

$\Psi(x, y ; q, a, \Phi)=\frac{\Psi\left(x, y ; \chi_{0}, \Phi\right)}{\phi(q)}+O\left(\frac{1}{\phi(q)} \sum_{0 \leq k \leq \log q / 2} e^{C_{2} k} \max _{\chi \in \Xi(k)}\left|\int_{\alpha-i \infty}^{\alpha+i \infty} L(s, \chi ; y) x^{s} \breve{\Phi}(s) d s\right|\right)$.

To prove Theorem 1, we will show that the "big Oh" term in the preceding equation is of smaller order than our lower bound for the main term. To this end we will separate the summation over $k$ into summations over different ranges, as described in $\S 2.2$. We see immediately, however, that

$\left|\int_{\alpha+i(y q)^{1 / 4}}^{\alpha+i \infty} L(s, \chi ; y) x^{s} \breve{\Phi}(s) d s\right| \leq L\left(\alpha, \chi_{0} ; y\right) x^{\alpha} \int_{\alpha+i(y q)^{1 / 4}}^{\alpha+i \infty}|\breve{\Phi}(s)| d s \ll \frac{L\left(\alpha, \chi_{0} ; y\right) x^{\alpha} \breve{\Phi}(\alpha)}{\breve{\Phi}(\alpha) y^{2} q^{2}}$

because of the rapid decay of $\breve{\Phi}(s)$. This is of smaller order than $\Psi\left(x, y ; \chi_{0}, \Phi\right) / y q^{2}$, (using the lower bound $\breve{\Phi}(\alpha) \geq 1 /(2 \alpha) \gg(\log x \log y) / y$ if $y \leq \log x)$, and clearly the same holds for the integral over $\left(\alpha-i \infty, \alpha-i(y q)^{1 / 4}\right]$. Thus, unless $y$ is very small (for which see $\S 2.7)$, it will suffice to prove satisfactory bounds for $\int_{\alpha-i(y q)^{1 / 4}}^{\alpha+i(y)^{1 / 4}} L(s, \chi ; y) x^{s} \breve{\Phi}(s) d s$, for $\chi \in \Xi(k)$. Note that $(y q)^{1 / 4} \leq q^{3 / 4}$ on our assumption that $q \geq \sqrt{y}$.

2.2. Ranges of $k$. For "large" $y$, in the sense of the introduction, we separate the summation over $0 \leq k \leq(\log q) / 2$ into three different ranges, as follows:

- the "basic range", $\sqrt{u} \leq k \leq(\log q) / 2$;

- the "Rodosskiul range", $4 A \log A+D \leq k<\sqrt{u}$, where $D$ is the absolute constant appearing in Rodosskiu Bound 1 in $\S 2.4$;

- the "problem range", $0 \leq k<4 A \log A+D$.

(In Theorem 1 we have $A=4 \sqrt{e}-\delta$, and the reader may think of $A$ simply as $O(1)$. We continue to explicitly record dependence on $A$ to aid anyone checking Theorem 2.)

This is analogous to Soundararajan's argument [8], but our definitions of the ranges are different. In $\S \S 2.3-2.5$ we study these ranges in turn, and the reader may compare with $\S \S 3-5$ of Soundararajan's article [8].

For smaller $y$ the situation is simpler because one can treat the "basic range" and the "Rodosskil range" in a unified way. This is discussed in $§ \S 2.6-2.7$. 
2.3. A modified zero-free region argument. In $\S 3$ we will prove the following result, which we will need in place of Lemma 3.2 of Soundararajan [8]:

Proposition 1. Let $B>0$ be fixed, and let $y \geq 2$ and $\sqrt{y} \leq q \leq y^{A}$. If $\log x /((B+$ 1) $\log q)$ and $k /((A+1) \log (u \log u))$ are larger than certain absolute constants, and if $y$ is "large" then the following holds. For any $\chi \in \Xi(k)$, any $\alpha-B k / \log x \leq \sigma \leq \alpha$, and any $|t| \leq q / 2$, we have

$$
|\log L(\sigma+i t, \chi ; y)-\log L(\alpha+i t, \chi ; y)| \leq k / 50+O(1)
$$

The proof of this involves using a smoothed explicit formula to analyse the first and second derivatives of $\log L(\sigma+i t, \chi ; y)$.

We will also use the following consequence of Fubini's theorem and the CauchySchwarz inequality, whose proof is an easy exercise:

Lemma 1. Suppose that $\beta, r>0$, and that $F(s)$ is any integrable function on the interval $[\beta, \beta+i r] \subseteq \mathbb{C}$. Let $G(s)$ be an Euler product of the following form:

$$
G(s):=\prod_{p \leq y}\left(1-\frac{g(p)}{p^{s}}\right)^{-1}
$$

where $y \geq 0$ is fixed, and $|g(p)| \leq 1$ for all primes $p$. Then

$$
\left|\int_{\beta}^{\beta+i r} G(s) F(s) d s\right| \leq M\left(|G(\beta)|+\sqrt{\left.\int_{\beta}^{\beta+i r}\left|\frac{G^{\prime}}{G}(s)\right|^{2} d|s| \int_{\beta}^{\beta+i r}|G(s)|^{2} d|s|\right)},\right.
$$

where

$$
M=M(\beta, r, F):=\sup _{0 \leq t \leq r}\left|\int_{\beta+i t}^{\beta+i r} F(s) d s\right| .
$$

We apply Proposition 1 with $B$ chosen as $C_{2}+2$, where $C_{2}$ is the constant in the log-free zero density estimate in $\S 2.1$. Using this together with the rapid decay of $\breve{\Phi}(s)$, we note firstly that, under the conditions of Proposition 1,

$$
\begin{aligned}
& \int_{\alpha-i(y q)^{1 / 4}}^{\alpha+i(y q)^{1 / 4}} L(s, \chi ; y) x^{s} \breve{\Phi}(s) d s \\
= & \int_{\alpha-\frac{B k}{\log x}-i \log ^{1 / 4} y}^{\alpha-\frac{B k}{\log x}+i \log ^{1 / 4} y} L(s, \chi ; y) x^{s} \breve{\Phi}(s) d s+\int_{\alpha-\frac{B k}{\log x}-i(y q)^{1 / 4}}^{\alpha-\frac{B k}{\log x}-i \log ^{1 / 4} y} L(s, \chi ; y) x^{s} \breve{\Phi}(s) d s+ \\
& +\int_{\alpha-\frac{B k}{\log x}+i \log ^{1 / 4} y}^{\alpha-\frac{B k}{\log x}+i(y q)^{1 / 4}} L(s, \chi ; y) x^{s} \breve{\Phi}(s) d s+O\left(e^{k / 50} L\left(\alpha, \chi_{0} ; y\right) x^{\alpha} / y^{2} q^{2}\right) .
\end{aligned}
$$

The second and third integrals may also be estimated just using Proposition 1, showing they are $O\left(e^{k / 50} L\left(\alpha, \chi_{0} ; y\right) x^{\alpha-B k / \log x} / \log ^{2} y\right)$. Both "big Oh" terms are

$$
\ll L\left(\alpha, \chi_{0} ; y\right) x^{\alpha}\left(\frac{1}{y^{2} q^{199 / 100}}+\frac{e^{-\left(C_{2}+99 / 50\right) k} \sqrt{u}}{\sqrt{\log x} \log ^{3 / 2} y}\right) \ll \Psi\left(x, y ; \chi_{0}, \Phi\right)\left(\frac{1}{y q^{199 / 100}}+\frac{e^{-\left(C_{2}+1\right) k}}{\log y}\right),
$$


on recalling our lower bound for $\Psi\left(x, y ; \chi_{0}, \Phi\right)$ and that $\log (u \log u) \ll k \leq(\log q) / 2$.

Combining Lemma 1 (with the choices $F(s)=x^{s} \breve{\Phi}(s)$ and $G(s)=L(s, \chi ; y)$ ) with Proposition 1, we see the first integral is

$$
\begin{aligned}
& \ll \sup _{0 \leq t \leq \log ^{1 / 4} y}\left|\int_{\alpha-\frac{B k}{\log x}+i t}^{\alpha-\frac{B k}{\log x}+i \log ^{1 / 4} y} x^{s} \breve{\Phi}(s) d s\right| \cdot\left(\left|L\left(\alpha-\frac{B k}{\log x}, \chi ; y\right)\right|+\right. \\
& +\sqrt{\left.\int_{\alpha-\frac{B k}{\log x}-i \log ^{1 / 4} y}^{\alpha-\frac{B k}{\log x}+i \log ^{1 / 4} y}\left|\frac{L^{\prime}(s, \chi ; y)}{L(s, \chi ; y)}\right|^{2} d|s| \int_{\alpha-\frac{B k}{\log x}-i \log ^{1 / 4} y}^{\alpha-\frac{B k}{\log x}+i \log ^{1 / 4} y}|L(s, \chi ; y)|^{2} d|s|\right)} \\
& \ll e^{-B k+k / 50} x^{\alpha} \sup _{0 \leq t \leq \log ^{1 / 4} y}\left|\int_{t}^{\log ^{1 / 4} y} x^{i r} \breve{\Phi}\left(\alpha-\frac{B k}{\log x}+i r\right) d r\right| \\
& \cdot\left(L\left(\alpha, \chi_{0} ; y\right)+\sqrt{\left.\int_{\alpha-\frac{B k}{\log x}-i \log ^{1 / 4} y}^{\alpha-\frac{B k}{\log x}+i \log ^{1 / 4} y}\left|\frac{L^{\prime}(s, \chi ; y)}{L(s, \chi ; y)}\right|{ }^{2} d|s| \int_{\alpha-i \log ^{1 / 4} y}^{\alpha+i \log ^{1 / 4} y}|L(s, \chi ; y)|^{2} d|s|\right) .}\right.
\end{aligned}
$$

At this point we invoke the following majorant principle for trigonometric sums, which we quote from chapter 7.3 of Montgomery's book [6]:

Majorant Principle 1 (Wirsing, and others). Let $\lambda_{1}, \ldots, \lambda_{N}$ be real numbers, and suppose that $\left|a_{n}\right| \leq A_{n}$ for all $n$. Then

$$
\int_{-T}^{T}\left|\sum_{n=1}^{N} a_{n} e^{2 \pi i \lambda_{n} t}\right|^{2} d t \leq 3 \int_{-T}^{T}\left|\sum_{n=1}^{N} A_{n} e^{2 \pi i \lambda_{n} t}\right|^{2} d t .
$$

The point is that $L(s, \chi ; y)$ and its logarithmic derivative $L^{\prime}(s, \chi ; y) / L(s, \chi ; y)$ are Dirichlet series, so in particular are trigonometric series with $\lambda_{n}$ chosen as $-\log n / 2 \pi$. (The majorant principle is stated for finite sums, but it remains valid for uniformly convergent Dirichlet series, as Montgomery [6] remarks.3.) Thus it will suffice to estimate the above with the squareroot term replaced by

$$
\sqrt{\int_{\alpha-\frac{B k}{\log x}-i \log ^{1 / 4} y}^{\alpha-\frac{B k}{\log x}+i \log ^{1 / 4} y}\left|\frac{L^{\prime}(s, 1 ; y)}{L(s, 1 ; y)}\right|^{2} d|s| \int_{\alpha-i \log ^{1 / 4} y}^{\alpha+i \log ^{1 / 4} y}\left|L\left(s, \chi_{0} ; y\right)\right|^{2} d|s| .}
$$

In our second appendix we show how to estimate the remaining integrals, which is fairly standard. It turns out, provided $y$ is "large" and $B k / \log x \leq 1 / 8$, say, (so $\alpha-B k / \log x \geq 3 / 4)$, that the whole of the above is $\ll e^{-B k+k / 50} x^{\alpha} \cdot \frac{L\left(\alpha, \chi_{0} ; y\right)}{\log x}\left(1+\sqrt{y^{2 B k / \log x} u^{2} \log u}\right) \ll \Psi\left(x, y ; \chi_{0}, \Phi\right) \sqrt{u \log u} e^{-\left(C_{2}+99 / 50-B / u\right) k}$. Thus if $u /(A+1)^{3}$ is larger than an absolute constant, (so $\sqrt{u} /((A+1) \log (u \log u))$ is large, and therefore Proposition 1 is applicable for $k$ in the "basic range"), we have the

\footnotetext{
${ }^{3}$ See Chapter III.4.3 of Tenenbaum [9] for an application of Majorant Principle 1 to Dirichlet series, concerning means of multiplicative functions. The author thanks K. Soundararajan for this reference.
} 
more than satisfactory estimate

$$
\left|\int_{\alpha-i(y q)^{1 / 4}}^{\alpha+i(y q)^{1 / 4}} L(s, \chi ; y) x^{s} \breve{\Phi}(s) d s\right| \ll \Psi\left(x, y ; \chi_{0}, \Phi\right)\left(\frac{1}{y q^{199 / 100}}+e^{-\left(C_{2}+1\right) k}\right)
$$

when $k$ is in that ranget.

2.4. A modified Rodosskiı argument. We modify the "Rodosskil type argument" from Soundararajan's paper [8] (in which zeros of $L$-series are studied with carefully chosen weights) in the same kind of way as the zero-free region argument, by using Fubini's theorem and Majorant Principle 1. We begin with a variant of Lemma 1.

Lemma 2. Suppose that $\beta, r, F(s), G(s)$ are as in the statement of Lemma 1. Then

$$
\left|\int_{\beta}^{\beta+i r} G(s) F(s) d s\right| \leq M^{*}\left(\left|G^{*}(\beta)\right|+\sqrt{\left.\int_{\beta}^{\beta+i r}\left|\frac{G^{\prime}}{G}(s)\right|^{2} d|s| \int_{\beta}^{\beta+i r}\left|G^{*}(s)\right|^{2} d|s|\right)},\right.
$$

where

$G^{*}(s):=\prod_{p \leq \sqrt{y}}\left(1-\frac{g(p)}{p^{s}}\right)^{-1}$, and $M^{*}:=\sup _{0 \leq t \leq r}\left(\left|\int_{\beta+i t}^{\beta+i r} F(s) d s\right| \prod_{\sqrt{y}<p \leq y}\left|1-\frac{g(p)}{p^{\beta+i t}}\right|^{-1}\right)$.

Similarly to $\S 2.3$, the rapid decay of $\breve{\Phi}(s)$ implies that

$$
\begin{aligned}
& \int_{\alpha-i(y q)^{1 / 4}}^{\alpha+i(y q)^{1 / 4}} L(s, \chi ; y) x^{s} \breve{\Phi}(s) d s \\
= & \int_{\alpha-i \log ^{1 / 4} y}^{\alpha+i \log ^{1 / 4} y} L(s, \chi ; y) x^{s} \breve{\Phi}(s) d s+O\left(\frac{x^{\alpha} L\left(\alpha, \chi_{0} ; \sqrt{y}\right)}{\log ^{2} y} \sup _{\log ^{1 / 4} y<|t| \leq(y q)^{1 / 4}} \prod_{\sqrt{y}<p \leq y}\left|1-\frac{\chi(p)}{p^{\alpha+i t}}\right|^{-1}\right) .
\end{aligned}
$$

Combining Lemma 2 with Majorant Principle 1, for "large" $y$ we see

$$
\begin{aligned}
& \int_{\alpha-i \log ^{1 / 4} y}^{\alpha+i \log ^{1 / 4} y} L(s, \chi ; y) x^{s} \breve{\Phi}(s) d s \\
\ll & \sup _{0 \leq t \leq \log ^{1 / 4} y}\left|\int_{\alpha+i t}^{\alpha+i \log ^{1 / 4} y} x^{s} \breve{\Phi}(s) d s\right| \cdot \sup _{|t| \leq \log ^{1 / 4} y} \prod_{\sqrt{y}<p \leq y}\left|1-\frac{\chi(p)}{p^{\alpha+i t}}\right|^{-1} \\
& \cdot\left(L\left(\alpha, \chi_{0} ; \sqrt{y}\right)+\sqrt{\left.\int_{\alpha-i \log ^{1 / 4} y}^{\alpha+i \log ^{1 / 4} y}\left|\frac{L^{\prime}(s, 1 ; y)}{L(s, 1 ; y)}\right|^{2} d|s| \int_{\alpha-i \log ^{1 / 4} y}^{\alpha+i \log ^{1 / 4} y}\left|L\left(s, \chi_{0} ; \sqrt{y}\right)\right|^{2} d|s|\right)}\right. \\
\ll & \frac{x^{\alpha} L\left(\alpha, \chi_{0} ; \sqrt{y}\right) u \sqrt{\log u}}{\log x} \sup _{|t| \leq \log ^{1 / 4} y} \prod_{\sqrt{y}<p \leq y}\left|1-\frac{\chi(p)}{p^{\alpha+i t}}\right|^{-1} .
\end{aligned}
$$

Here the final inequality again used the estimates from our second appendix.

\footnotetext{
${ }^{4}$ We could use our argument on a much wider range of $k$ than the "basic range". However, this would not quite be large enough to dispense with the "Rodosskiı̌ range" argument in $\S 2.4$, and splitting into ranges as we do yields better quantitative bounds on our integrals.
} 
Obtaining a non-trivial bound has now reduced to obtaining a sufficiently non-trivial estimate for the products over primes. This will follow from the next result, which is the content of Lemmas 4.2 and 4.3 of Soundararajan [8].

Rodosskiu Bound 1 (Soundararajan, 2008). There is an absolute constant $D$ for which the following is true. Suppose that $\chi \in \Xi(k)$ for some $k \geq 4 A \log A+D$. If $q \leq y^{A}$, and $|t| \leq q / 2$, and $y /(A+1)^{2}$ is large enough, then

$$
\sum_{\sqrt{y} \leq p \leq y, p \nmid q} \frac{1-\Re\left(\chi(p) p^{-i t}\right)}{p} \log p \geq \frac{\log y}{5} .
$$

Then

$$
\left|\frac{1-\chi(p) p^{-\alpha-i t}}{1-p^{-\alpha}}\right|=\left|1+\frac{1-\chi(p) p^{-i t}}{p^{\alpha}-1}\right| \geq 1+\sum_{k=1}^{\infty} \frac{1-\Re\left(\chi(p) p^{-i t}\right)}{p^{k \alpha}} \geq e^{\left(1-\Re\left(\chi(p) p^{-i t}\right)\right) / p^{\alpha}},
$$

so (as in the argument of Lemma 4.2 of Soundararajan [8]) we find

$$
\begin{aligned}
\frac{L\left(\alpha, \chi_{0} ; \sqrt{y}\right)}{L\left(\alpha, \chi_{0} ; y\right)} \sup _{|t| \leq(y q)^{1 / 4}} \prod_{\sqrt{y}<p \leq y}\left|1-\frac{\chi(p)}{p^{\alpha+i t}}\right|^{-1} & =\sup _{|t| \leq(y q)^{1 / 4}} \prod_{\sqrt{y}<p \leq y}\left|\frac{1-\chi(p) p^{-\alpha-i t}}{1-\chi_{0}(p) p^{-\alpha}}\right|^{-1} \\
& \leq \sup _{|t| \leq(y q)^{1 / 4}} e^{-\sum_{\sqrt{y}<p \leq y, p \nmid q}\left(1-\Re\left(\chi(p) p^{-i t}\right)\right) / p^{\alpha}} \\
& \ll e^{-y^{(1-\alpha) / 2} / 5} \\
& \ll e^{-\Theta(\sqrt{u \log u})} .
\end{aligned}
$$

We conclude that, under the conditions of Rodosskiu Bound 1 (and for "large" y),

$$
\left|\int_{\alpha-i(y q)^{1 / 4}}^{\alpha+i(y q)^{1 / 4}} L(s, \chi ; y) x^{s} \breve{\Phi}(s) d s\right| \ll \sqrt{u \log u} e^{-\Theta(\sqrt{u \log u})} \Psi\left(x, y ; \chi_{0}, \Phi\right) .
$$

This estimate more than suffices for $\chi \in \Xi(k)$ with $k$ in the "Rodosskiu range".

2.5. The remaining characters. It remains to analyse $\Psi(x, y ; \chi, \Phi)$ when $\chi \in \mathcal{A}:=$ $\bigcup_{k<4 A \log A+D} \Xi(k)$. Soundararajan's arguments work reasonably for this, the only adaptations being of the kind that we have demonstrated in $\S \S 2.3-2.4$, so a brief discussion seems sufficient.

We write $B=B(A):=\# \mathcal{A}$, which is bounded in terms of $A$ and $D$ because of the log-free zero density estimate (recall $§ 2.1$ ). The following result (which is proved by considering the corresponding sums with $\chi(p) p^{-i t}$ replaced by $\chi(p)^{k} p^{-i t k}$, for $1 \leq k \leq$ $B+1$ ) is the content of Proposition 5.1 of Soundararajan [8]: 
Rodosskiu Bound 2 (Soundararajan, 2008). Suppose that $\chi$ is a character of order exceeding $B=B(A)$. If $q \leq y^{A}$, and $|t| \leq q /(2(B+1))$, and $y /(A+1)^{2}$ is large, then

$$
\sum_{\sqrt{y} \leq p \leq y, p \nmid q} \frac{1-\Re\left(\chi(p) p^{-i t}\right)}{p} \log p \geq \frac{\log y}{5(B+1)^{2}} .
$$

Using Rodosskiu Bound 2 in place of Rodosskil Bound 1, one can proceed as in $\S 2.4$ to bound $\Psi(x, y ; \chi, \Phi)$ for characters $\chi$ of order at least $B+1$ (with $e^{-\Theta\left(\sqrt{u \log u} /(B+1)^{2}\right)}$ ultimately replacing $e^{-\Theta(\sqrt{u \log u})}$ in the estimates $)$.

It now remains to treat $\chi \in \mathcal{B}$, where $\mathcal{B}:=\mathcal{A} \cap\{\chi: \chi$ has order $\leq B\}$. To simplify our formulae, we temporarily set $g=\log u / \sqrt{\log x \log y}=\log u /(\sqrt{u} \log y)$. A very small adaptation of the argument in $\S 2.4$ yields

$$
\begin{aligned}
& \left|\int_{\alpha-i(y q)^{1 / 4}}^{\alpha+i(y q)^{1 / 4}} L(s, \chi ; y) x^{s} \breve{\Phi}(s) d s\right| \\
\ll & \left|\int_{\alpha-i g}^{\alpha+i g} L(s, \chi ; y) x^{s} \breve{\Phi}(s) d s\right|+\sqrt{u \log u} \Psi\left(x, y ; \chi_{0}, \Phi\right) \sup _{g<|t| \leq(y q)^{1 / 4}} e^{-\sum_{\sqrt{y}<p \leq y} \frac{\left(1-\Re\left(\chi(p) p^{-i t}\right)\right)}{p^{\alpha}}} \\
\ll & \frac{x^{\alpha} \breve{\Phi}(\alpha) \log u}{\sqrt{\log x \log y}} L\left(\alpha, \chi_{0} ; y\right) \sup _{|t| \leq g} e^{-\sum_{p \leq y, p \nmid q} \frac{\left(1-\Re\left(\chi(p) p^{-i t}\right)\right)}{p^{\alpha}}}+ \\
& +\sqrt{u \log u} \Psi\left(x, y ; \chi_{0}, \Phi\right) \sup _{g<|t| \leq(y q)^{1 / 4}} e^{-\sum_{\sqrt{y}<p \leq y, p \nmid q} \frac{\left(1-\Re\left(\chi(p) p^{-i t}\right)\right)}{p^{\alpha}}},
\end{aligned}
$$

if $y$ is "large". By the argument of Lemma 5.2 of Soundararajan [8], (which is proved by a neat reduction from working with characters of order at most $B(A)$ to working with the principal character), provided that $u \geq B^{3}$ (say) the exponential in the second term is $\ll e^{-\Theta\left(\log ^{2} u\right)}$. This gives an acceptable bound for that term.

Finally we apply the following result, which may be extracted 5 from Lemmas 5.2 and 5.3 of Soundararajan [8].

Character Sum Bound 1 (Soundararajan, 2008). Let $\delta>0$ be sufficiently small, and suppose that $q \leq y^{4 \sqrt{e}-20 \delta}$. If $\chi \neq \chi_{0}$ is a character of order at most $B$, and if $|t| \leq 1 /(B \log y)$, then

$$
\sum_{p \leq y, p \nmid q} \frac{1-\Re\left(\chi(p) p^{-i t}\right)}{p^{\alpha}} \gg \frac{1}{B} \log \left(\left(\delta+O\left(\frac{1}{\log y}\right)+O_{\delta}\left(\frac{B^{3 \delta / 2} \log ^{2} y}{y^{\delta^{2}}}\right)\right) y^{\delta(1-\alpha) / 2}\right)+O(1) .
$$

${ }^{5}$ It requires a little care to obtain the explicit error terms in Character Sum Bound 1. The reader should note that if $\chi$ is a (primitive) non-principal character to modulus $q$, of order $l$, and $\delta>0$ is small, then Heath-Brown's [4] refined character sum estimate shows in particular that

$$
\sum_{n \leq H} \chi(n) \ll_{\delta} H l^{3 \delta / 2}\left(q^{1 / 4} H^{-1}\right)^{\delta} q^{\delta^{2} / 2}
$$

This should be applied with $H=z / d \geq y^{\sqrt{e}-3 \delta / 2} \geq q^{1 / 4} y^{7 \delta / 2}$ in the proof of Soundararajan's 8 ] Lemma 5.3. 
Using this to bound the exponential in the first term, we complete the proof of Theorem 1 for "large" values of $y$. Character Sum Bound 1 imports Burgess's character sum estimates (with the modification of Heath-Brown [4] for characters of bounded order): indeed, it is clear that when $|t|$ is so small, cancellation in $L(\alpha+i t, \chi ; y)$ amounts to cancellation in sums of $\chi$. If one could prove non-trivial bounds for shorter character sums, one could introduce them at this point and thereby extend the range of $q$ in Theorem 1. These remarks apply equally to Soundararajan's proof [8] on the range of $y$ where it is valid.

2.6. The case of small $y$. When $y$ is "small" the argument from Soundararajan's paper [8] almost goes through, even in a simplified form. The complication is that the parameter $\alpha(x, y)$ behaves differently when $y \leq \log x$ than otherwise, and in particular is very small, which necessitates a few changes. We sketch these briefly.

Thus when $y \leq \log x$, we have

$$
\begin{aligned}
\left|\frac{L(\alpha+i t, \chi ; y)}{L\left(\alpha, \chi_{0} ; y\right)}\right|=\prod_{p \leq y, p \nmid q}\left|1+\frac{1-\chi(p) p^{-i t}}{p^{\alpha}-1}\right|^{-1} & \leq \prod_{p \leq y, p \nmid q}\left|1+\frac{1-\Re\left(\chi(p) p^{-i t}\right)}{y^{\alpha}-1}\right|^{-1} \\
& \leq \prod_{p \leq y, p \nmid q}\left|1+\frac{c \log x\left(1-\Re\left(\chi(p) p^{-i t}\right)\right)}{y}\right|^{-1}
\end{aligned}
$$

for a small constant $c>0$, since $\alpha(x, y)=O(y / \log x \log y)$ for $y \leq \log x$. By calculus, $1+c t \geq(1+t)^{c}$ for $t \geq 0$ and $0 \leq c \leq 1$, so the above is at most

$$
\left(1+\frac{\log x}{y}\right)^{-c \sum_{p \leq y, p \nmid q}\left(1-\Re\left(\chi(p) p^{-i t}\right)\right)} \leq\left(1+\frac{\log x}{y}\right)^{-c \frac{\sqrt{y}}{\log y} \sum_{\sqrt{y} \leq p \leq y, p \nmid q} \frac{1-\Re\left(\chi(p) p^{-i t}\right)}{p} \log p} .
$$

It follows, using the decay of $\breve{\Phi}$ to control the large range of integration, that

$$
\begin{aligned}
& \left|\int_{\alpha-i(y q)^{1 / 4}}^{\alpha+i(y q)^{1 / 4}} L(s, \chi ; y) x^{s} \breve{\Phi}(s) d s\right| \\
\ll & x^{\alpha} \breve{\Phi}(\alpha) L\left(\alpha, \chi_{0} ; y\right) \sup _{|t| \leq(y q)^{1 / 4}}\left(1+\frac{\log x}{y}\right)^{-c \frac{\sqrt{y}}{\log y} \sum_{\sqrt{y} \leq p \leq y, p \nmid q} \frac{1-\Re\left(\chi(p) p^{-i t}\right)}{p} \log p} .
\end{aligned}
$$

If $\chi \in \Xi(k)$ for some $k \geq 4 A \log A+D$ then Rodosskiu Bound 1 shows the above is $O\left(\Psi\left(x, y ; \chi_{0}, \Phi\right) /\left(\log x+2^{y^{1 / 3}}\right)\right)$, say (since we assume $\left.1 \ll y \leq \log x\right)$.

When $\log x<y \leq e^{\log ^{1 / 10} x}$, one can argue instead (as in $\S 2.4$ ) that

$$
\left|\frac{L(\alpha+i t, \chi ; y)}{L\left(\alpha, \chi_{0} ; y\right)}\right| \leq \prod_{p \leq y, p \nmid q}\left(1+\sum_{k=1}^{\infty} \frac{1-\Re\left(\chi(p) p^{-i t}\right)}{p^{k \alpha}}\right)^{-1} \leq e^{-\frac{y^{(1-\alpha) / 2}}{\log y} \sum_{\sqrt{y} \leq p \leq y, p \nmid q} \frac{1-\Re\left(\chi(p) p^{-i t}\right)}{p} \log p},
$$


so Rodosskiu Bound 1 implies that the integral is $O\left(\Psi\left(x, y ; \chi_{0}, \Phi\right) / 2^{\log ^{4} y}\right)$, say $\left(\right.$ as $y^{1-\alpha} \gg$ $\left.u \geq \log ^{9 / 10} x \geq \log ^{9} y\right)$. These estimates are more than acceptable for $\chi \in \Xi(k)$, $4 A \log A+D \leq k \leq(\log q) / 2$.

We must still deal with $\chi \in \Xi(k)$ for $k$ in the "problem range". As in $\S 2.5$, if $\chi$ has order at least $B(A)+1$ then we need only apply Rodosskil Bound 2 instead of Rodosskil Bound 1 in the preceding calculations. If $\chi \in \mathcal{B}$ we set $h=$ $\log u / \sqrt{\min \{\log x \log y, y \log y\}}$, so $\left|\int_{\alpha-i(y q)^{1 / 4}}^{\alpha+i(y)^{1 / 4}} L(s, \chi ; y) x^{s} \breve{\Phi}(s) d s\right|$ is

$$
\ll x^{\alpha} \breve{\Phi}(\alpha)\left(h \sup _{|t| \leq h}|L(\alpha+i t, \chi ; y)|+L\left(\alpha, \chi_{0} ; y\right) \sup _{h<|t| \leq(y q)^{1 / 4}} e^{-c \sum_{p \leq y, p \nmid q} \frac{\left(1-\Re\left(\chi(p) p^{-i t}\right)\right)}{p^{\alpha}}}\right) .
$$

Provided that $u \geq B^{3}$ and $y \geq B^{7}$ (say), the argument of Lemma 5.2 of Soundararajan [8] shows the exponential is $\ll e^{-\Theta\left(\log ^{2} u\right)}+e^{-\Theta\left(y^{2 / 3}\right)}$, which is $\ll e^{-\Theta\left((\log \log x)^{2}\right)}$ for "small" $y$ (that is $\left.(\log \log x)^{3} \leq y \leq e^{\log ^{1 / 10} x}\right)$. This is also acceptable.

Finally we apply Character Sum Bound 1 to estimate $\sup _{|t| \leq h}|L(\alpha+i t, \chi ; y)|$. If $y \geq \log x$ this works precisely as in $\S 2.5$. If $(\log \log x)^{3} \leq y \leq \log x$ we have

$$
\sup _{|t| \leq h}|L(\alpha+i t, \chi ; y)| \leq\left(1+\frac{\log x}{y}\right)^{-(c / B) \log \left((\delta+o(1)) y^{\delta(1-\alpha) / 2}\right)+O(1)} L\left(\alpha, \chi_{0} ; y\right),
$$

noting that $h \leq 1 / \log ^{2} y$ (say) for such $y$, so Character Sum Bound 1 is applicable. Now $x^{\alpha} \breve{\Phi}(\alpha) h L\left(\alpha, \chi_{0} ; y\right) \ll\left(\Psi\left(x, y ; \chi_{0}, \Phi\right) \log u \log x\right) / y$ when $y \leq \log x$, and that is $\ll \Psi\left(x, y ; \chi_{0}, \Phi\right) \log y((\log x) / y)^{2}$. This all implies an acceptable bound for $x^{\alpha} \breve{\Phi}(\alpha) h \sup _{|t| \leq h}|L(\alpha+i t, \chi ; y)|$, finishing the proof of Theorem 1 for "small" $y$.

2.7. The case of very small $y$. If $y$ is "very small", two changes are needed to the argument for "small" $y$ in $\S 2.6$ (and at the end of $\S 2.1$ ).

Firstly, if $q \sqrt{y} \leq(\log \log x)^{1 / 3}$ we cannot bound integrals like $\int_{\alpha+i(y q)^{1 / 4}}^{\alpha+i \infty} L(s, \chi ; y) x^{s} \breve{\Phi}(s) d s$ acceptably just using the decay of $\breve{\Phi}$, necessarily. As an alternative, if $y \leq \sqrt{\log x}$, if $p_{0} \leq y$ is any prime not dividing $q$, and if $\epsilon>0$, then as in $\S 2.6$ we have

$$
\begin{aligned}
& \left|\int_{|t| \geq \epsilon} L(\alpha+i t, \chi ; y) x^{\alpha+i t} \breve{\Phi}(\alpha+i t) d t\right| \\
\ll & x^{\alpha} L\left(\alpha, \chi_{0} ; y\right) \int_{|t| \geq \epsilon}\left(1+\frac{\log x}{y}\right)^{-c\left(1-\Re\left(\chi\left(p_{0}\right) p_{0}^{-i t}\right)\right)} \frac{1}{|t|(|t|+1)^{8}} d t \\
\ll & \Psi\left(x, y ; \chi_{0}, \Phi\right) \sqrt{\frac{y}{\log y}} \int_{|t| \geq \epsilon}(\log x)^{-c\left(1-\Re\left(\chi\left(p_{0}\right) p_{0}^{-i t}\right)\right) / 2} \frac{1}{|t|(|t|+1)^{8}} d t,
\end{aligned}
$$

on recalling the lower bound for $\Psi\left(x, y ; \chi_{0}, \Phi\right)$ (and $\breve{\Phi}(\alpha)$ ) from $\S 2.1$. But

$$
1-\Re\left(\chi\left(p_{0}\right) p_{0}^{-i t}\right)=1-\cos \left(\arg \left(\chi\left(p_{0}\right)\right)-t \log p_{0}\right),
$$


which is clearly $\geq 1 /(\log \log x)^{4 / 5}$, say, except on a progression of intervals of $t$ having lengths $\Theta\left(1 /\left(\log p_{0}(\log \log x)^{2 / 5}\right)\right)$ and spacing $\Theta\left(1 / \log p_{0}\right)$. Thus if $\epsilon \geq 1 /(\log \log x)^{2 / 5-1 / 3}$, the integral is

$$
\ll \Psi\left(x, y ; \chi_{0}, \Phi\right) \sqrt{\frac{y}{\log y}} \frac{1}{\epsilon}\left(\frac{1}{(\log \log x)^{2 / 5}}+e^{-\Theta\left((\log \log x)^{1 / 5}\right)}\right) \ll \frac{\sqrt{y} \Psi\left(x, y ; \chi_{0}, \Phi\right)}{(\log \log x)^{1 / 3}} .
$$

Note that this holds for all Dirichlet characters $\chi$ to modulus $q$. If $q \sqrt{y} \leq(\log \log x)^{1 / 3}$ then we apply this with $\epsilon$ chosen as $\min \{q /(2(B(A)+1)), 1\}$, (which is at least $1 / y^{2 / 5-1 / 3}$ by assumption that $y$ is large in terms of $A$, and thus at least $\left.1 /(\log \log x)^{2 / 5-1 / 3}\right)$. Then we can estimate $\int_{|t| \leq \epsilon} L(\alpha+i t, \chi ; y) x^{\alpha+i t} \breve{\Phi}(\alpha+i t) d t$ for $\chi \notin \mathcal{B}$ using the Rodosskiu Bounds, exactly as demonstrated in $§ 2.6$.

(Note that we need not assume that $q \geq \sqrt{y}$, as previously, for the Rodosskiu Bounds to apply, since we are concerned with $|t| \leq \epsilon \leq q /(2(B+1))$ rather than $|t| \leq(y q)^{1 / 4}$.)

Secondly, to deal with $\chi \in \mathcal{B}$ we just set $h=1 / \log ^{2} y$, rather than choosing $h$ as in $\S 2.6$. Then $\left|\int_{\alpha-i(y q)^{1 / 4}}^{\alpha+i(y q)^{1 / 4}} L(s, \chi ; y) x^{s} \breve{\Phi}(s) d s\right|$, or $\left|\int_{\alpha-i \epsilon}^{\alpha+i \epsilon} L(s, \chi ; y) x^{s} \breve{\Phi}(s) d s\right|$, is $\ll x^{\alpha} \breve{\Phi}(\alpha)\left(h \sup _{|t| \leq h}|L(\alpha+i t, \chi ; y)|+L\left(\alpha, \chi_{0} ; y\right) \sup _{h<|t| \leq(y q)^{1 / 4}}\left(1+\frac{\log x}{y}\right)^{-c \sum_{p \leq y, p \nmid q} \frac{\left(1-\Re\left(\chi(p) p^{-i t}\right)\right)}{p^{\alpha}}}\right)$, where these terms may be bounded as in $\S 2.6$. In particular the exponential is $\ll$ $(\log x)^{-\Theta\left(y^{2 / 3}\right)}$ if $B^{7} \leq y \leq(\log \log x)^{3}$, which is more than satisfactory.

Q.E.D.

\section{Proof of Proposition 1}

To start the proof of Proposition 1, as used in $§ 2.3$, we shall establish the following lemma. We will need the result, and the techniques of the proof will also be used again.

Lemma 3. Under the assumptions of Proposition 1, and for primitive $\chi$, we have

$$
\left|\frac{L^{\prime}(\sigma+i t, \chi)}{L(\sigma+i t, \chi)}\right| \ll \log q
$$

To see this, we note that the left hand side is certainly at most

$$
\left|\frac{L^{\prime}(1+1 / \log q+i t, \chi)}{L(1+1 / \log q+i t, \chi)}\right|+\left(1+\frac{1}{\log q}-\sigma\right) \sup _{\sigma \leq \sigma^{\prime} \leq 1+1 / \log q}\left|\frac{d}{d \sigma^{\prime}} \frac{L^{\prime}\left(\sigma^{\prime}+i t, \chi\right)}{L\left(\sigma^{\prime}+i t, \chi\right)}\right| .
$$

Here the first term is at most $\zeta^{\prime}(1+1 / \log q) / \zeta(1+1 / \log q)$, which is $O(\log q)$. We also note that

$$
1+1 / \log q-\sigma \leq(1-\alpha(x, y))+1 / \log q+B k / \log x \leq k /(4 \log q),
$$

say, in view of the assumptions on $y, q$ and $k$ in Proposition 1. 
For primitive $\chi$, differentiation of the explicit formula for $\frac{L^{\prime}(s, \chi)}{L(s, \chi)}$ (which is e.g. formula (17) in chapter 12 of Davenport [1]) yields

$$
\frac{d}{d \sigma^{\prime}} \frac{L^{\prime}\left(\sigma^{\prime}+i t, \chi\right)}{L\left(\sigma^{\prime}+i t, \chi\right)}=-\sum_{n=0}^{\infty} \frac{1}{\left(2 n+\sigma^{\prime}+i t+a(\chi)\right)^{2}}-\sum_{\rho} \frac{1}{\left(\sigma^{\prime}+i t-\rho\right)^{2}},
$$

where the second sum is over the non-trivial zeros of $L(s, \chi)$, and $a(\chi)$ is 0 or 1 according as $\chi(-1)$ is 1 or -1 . (Thus the first sum is really over the trivial zeros of $L(s, \chi)$ : see e.g. chapters 9 and 19 of Davenport [1]). The sum over $n$ is clearly $O(1)$, and since $\sigma^{\prime} \geq \sigma \geq 1-k /(4 \log q),|t| \leq q / 2$ and $\chi \in \Xi(k)$ we have

$$
\begin{aligned}
\sum_{\rho} \frac{1}{\left|\sigma^{\prime}+i t-\rho\right|^{2}} & \leq \sum_{\substack{|\Im(\rho)-t| \leq 1, \mid \Im(\rho) \leq q q}} \frac{1}{\left|\sigma^{\prime}+i t-\rho\right|^{2}}+\sum_{\substack{|\Im(\rho)-t|>1,|\Im(\rho)| \leq q}} \frac{1}{\left|\sigma^{\prime}+i t-\rho\right|^{2}}+\sum_{|\Im(\rho)|>q} \frac{4}{|\Im(\rho)|^{2}} \\
& \ll \sum_{|\Im(\rho)-t| \leq 1} \frac{1}{|1+1 / \log q+i t-\rho|^{2}}+\log q+\frac{\log q}{q},
\end{aligned}
$$

using the fact that $\Re(\rho) \leq 1-k / \log q$ in the first sum, and standard results on the vertical distribution of zeros of $L(s, \chi)$ (as in e.g. chapter 16 of Davenport [1]).

To bound the remaining sum, we again use the fact that $\chi \in \Xi(k)$, noting that

$$
\begin{aligned}
\sum_{|\Im(\rho)-t| \leq 1} \frac{1}{|1+1 / \log q+i t-\rho|^{2}} & =\sum_{|\Im(\rho)-t| \leq 1} \frac{1}{\Re(1+1 / \log q+i t-\rho)} \Re\left(\frac{1}{1+1 / \log q+i t-\rho}\right) \\
& \leq \frac{\log q}{k} \Re\left(\sum_{|\Im(\rho)-t| \leq 1} \frac{1}{1+1 / \log q+i t-\rho}\right) \\
& =\frac{\log q}{k} \Re\left(\frac{L^{\prime}(1+1 / \log q+i t, \chi)}{L(1+1 / \log q+i t, \chi)}+O(\log q)\right) \\
& \ll \frac{\log ^{2} q}{k} .
\end{aligned}
$$

Here the second equality is a classical approximation for $L^{\prime}(s, \chi) / L(s, \chi)$, as in formula (4) of chapter 16 of Davenport [1]. See section 4 of Soundararajan [8], and especially chapter 9.2 of Montgomery [6], for further illustration of this argument.

Combining the estimates we obtained proves Lemma 3.

Now we note that, under the conditions of Proposition 1,

$$
\begin{aligned}
|\log L(\sigma+i t, \chi ; y)-\log L(\alpha+i t, \chi ; y)| & \leq(\alpha-\sigma) \sup _{\sigma \leq \sigma^{\prime} \leq \alpha}\left|\frac{L^{\prime}\left(\sigma^{\prime}+i t, \chi ; y\right)}{L\left(\sigma^{\prime}+i t, \chi ; y\right)}\right| \\
& \leq \frac{B k}{\log x}\left(\sup _{\alpha-\frac{B k}{\log x} \leq \sigma^{\prime} \leq \alpha}\left|\sum_{n \leq y} \frac{\Lambda(n) \chi(n)}{n^{\sigma^{\prime}+i t}}\right|+O(1)\right) .
\end{aligned}
$$

We suppose initially that $\chi$ is a primitive Dirichlet character, and also that it will suffice to bound the above with the sum over $n$ replaced by $\sum_{n \leq R y} w(n) \frac{\Lambda(n) \chi(n)}{n^{\sigma^{\prime}+i t}}$, where 
$R:=\max \left\{2, y^{y^{-k / 2 \log q}}\right\}$ and

$$
w(n):= \begin{cases}1 & \text { if } 1 \leq n \leq y \\ 1-\frac{\log (n / y)}{\log R} & \text { if } y \leq n \leq R y \\ 0 & \text { otherwise }\end{cases}
$$

At the end of the argument we will show how to remove these assumptions.

Recall that for $\Re(s)>1$ we have $\sum_{n=1}^{\infty} \frac{\Lambda(n) \chi(n)}{n^{s}}=-\frac{L^{\prime}(s, \chi)}{L(s, \chi)}$. Then a fairly standard contour integration procedure, as in e.g. chapters 13.2 and 12.1 of Montgomery and Vaughan [7], reveals that

$$
\begin{aligned}
\sum_{n \leq R y} w(n) \frac{\Lambda(n) \chi(n)}{n^{s}}= & -\frac{L^{\prime}(s, \chi)}{L(s, \chi)}-\frac{1}{\log R} \sum_{n=0}^{\infty} \frac{(R y)^{-2 n-a(\chi)-s}-y^{-2 n-a(\chi)-s}}{(2 n+a(\chi)+s)^{2}}- \\
& -\frac{1}{\log R} \sum_{\rho} \frac{(R y)^{\rho-s}-y^{\rho-s}}{(\rho-s)^{2}}
\end{aligned}
$$

whenever $L(s, \chi) \neq 0$. Here our notation is exactly as above. As always, the purpose of introducing the smoother weight $w(n)$ was to obtain nicer behaviour in these sums, namely that all denominators are raised to at least the second power. This is also the reason that it was a good idea to switch to studying $\frac{d}{d \sigma^{\prime}} \frac{L^{\prime}\left(\sigma^{\prime}+i t, \chi\right)}{L\left(\sigma^{\prime}+i t, \chi\right)}$ in proving Lemma 3.

Putting $s=\sigma^{\prime}+i t$, Lemma 3 and a trivial estimation show the first two terms in the above are $O(\log q)$. To estimate the sum over $\rho$ we proceed as in the proof of Lemma 3 , noting that we can extract a power saving $O\left(y^{-3 k /(4 \log q)}\right)$ on the range $|\Im(\rho)| \leq q$ (since $\sigma^{\prime} \geq 1-k /(4 \log q)$ and $\left.\chi \in \Xi(k)\right)$. Thus we have

$$
\begin{aligned}
\left|\frac{1}{\log R} \sum_{\rho} \frac{(R y)^{\rho-\sigma^{\prime}-i t}-y^{\rho-\sigma^{\prime}-i t}}{\left(\rho-\sigma^{\prime}-i t\right)^{2}}\right| & \ll \frac{y^{-3 k /(4 \log q)} \log ^{2} q}{k \log R}+\frac{1}{\log R} \sum_{|\Im(\rho)|>q} \frac{(R y)^{k /(4 \log q)}}{|\Im(\rho)|^{2}} \\
& \ll \frac{A \log q}{k}+\frac{y^{1 / 4} \log q}{q},
\end{aligned}
$$

since $\log R \geq y^{-k /(2 \log q)} \log y$ but $R y \leq y^{2}$. This is acceptable for Proposition 1 .

It remains to justify the two assumptions that we made at the start of the proof. Firstly, if $k$ is such that $R=y^{y^{-k / 2 \log q}} \geq 2$ then

$$
\left|\sum_{y<n \leq R y} w(n) \frac{\Lambda(n) \chi(n)}{n^{\sigma^{\prime}+i t}}\right| \leq \sum_{y<n \leq R y} \frac{\Lambda(n)}{n^{\sigma^{\prime}}} \ll \frac{y^{1-\sigma^{\prime}}\left(R^{1-\sigma^{\prime}}-1\right)}{1-\sigma^{\prime}},
$$

and since $1-\sigma^{\prime} \leq k /(4 \log q)$ this is $\ll y^{1-\sigma^{\prime}} \log R$, which is at most $\log y$. If $k$ is such that $R=2$, the same argument produces a bound $O\left(y^{1-\sigma^{\prime}}\right)$, or alternatively

$$
\left|\sum_{y<n \leq 2 y} w(n) \frac{\Lambda(n) \chi(n)}{n^{\sigma^{\prime}+i t}}\right| \leq \frac{1}{y^{\sigma^{\prime}}} \max _{y<m \leq 2 y}\left|\sum_{y<n \leq m} \frac{\Lambda(n) \chi(n)}{n^{i t}}\right| \ll y^{1-\sigma^{\prime}-k / \log q} \log ^{2} q .
$$


Here the first inequality follows from Abel's partial summation lemma, and the second from Lemma 3.1 of Soundararajan [8] (or directly by an explicit formula argument rather easier than the above calculations). Comparing our two bounds, we see that when $R=2$ the sum must be $\ll y^{1-\sigma^{\prime}-k / 2 \log q} \log q$, which is at most $\log q$. These error estimates are acceptable for Proposition 1.

Finally, if $\chi$ is not primitive we can apply the above techniques to the primitive character inducing $\chi$. This results in an error term of size at most

$$
\sum_{p \mid q} \log p \sum_{r \geq 1} \frac{1}{p^{r \sigma^{\prime}}} \ll \sum_{p \mid q} \log p \leq \log q
$$

when estimating $\sum_{n \leq y} \frac{\Lambda(n) \chi(n)}{n^{\sigma^{\prime}+i t}}$, which again is acceptable for Proposition 1 .

Q.E.D.

\section{Appendix A. Unsmoothing}

In this appendix we briefly explain how to pass from results about $\Psi(x, y ; q, a, \Phi)$, which we actually proved, to results about the unsmoothed version $\Psi(x, y ; q, a)$.

To "unsmooth" one notes that if $\epsilon>0$, and $\mathbf{1}_{[0,1-\epsilon]} \leq \Phi \leq \mathbf{1}_{[0,1]}$, then

$$
\begin{aligned}
\Psi(x, y ; q, a) \geq \Psi(x, y ; q, a, \Phi) & =\frac{1}{\phi(q)} \Psi\left(x, y ; \chi_{0}, \Phi\right)\left(1+o_{\Phi}(1)\right) \\
& \geq \frac{1}{\phi(q)} \Psi_{q}(x, y)\left(1-\frac{\left(\Psi_{q}(x, y)-\Psi_{q}((1-\epsilon) x, y)\right)}{\Psi_{q}(x, y)}+o_{\Phi}(1)\right)
\end{aligned}
$$

where the first equality is what we proved in the body of this paper. One can similarly obtain an upper bound for $\Psi(x, y ; q, a)$, so to deduce Theorem 1 we need to know that for any $\eta>0$, the ratio $\left(\Psi_{q}(x, y)-\Psi_{q}((1-\epsilon) x, y)\right) / \Psi_{q}(x, y)$ will be at most $\eta$ if $\epsilon$ is chosen sufficiently small (and $\log x / \log q$ is large enough). This local result about $\Psi_{q}(x, y)$ follows from Théorème 2.4 of de la Bretèche and Tenenbaum [2], (also see Theorem 3 of Hildebrand and Tenenbaum [5]), except when $y$ does not tend to infinity with $x$. However, if $2 \leq y \leq \sqrt{\log x}$ one has

$$
\Psi_{q}(x, y)=\frac{1}{(\#\{p \leq y: p \text { is prime, } p \nmid q\}) !} \prod_{p \leq y, p \nmid q}\left(\frac{\log x}{\log p}\right)\left(1+O\left(\frac{y^{2}}{\log x \log y}\right)\right),
$$

exactly similarly to an expression for $\Psi(x, y)$ due to Ennola (and explained in Chapter III.5.2 of Tenenbaum's book [9]), which directly implies that $\Psi_{q}((1-\epsilon) x, y)$ is $(1+$ $O(\epsilon)) \Psi_{q}(x, y)$, say. Actually one obtains a bound $O(\epsilon)$ for our ratio in all cases, provided that $\log x / \log q$ is large enough in terms of $\epsilon$.

For the proof of Theorem 2, one should apply this procedure to $\Psi(x, y ; \chi, \Phi)$ for all of the (bounded number of) characters $\chi \in \mathcal{B}$. The "analytic" unsmoothing procedure used by Soundararajan [8] appears not to work on our extended range of $y$, because one 
cannot replace $\Psi(x, y ; \chi, \Phi)$ by a contour integral over a suitably short range of $t$ for $\breve{\Phi}$ to be removed from it.

\section{Appendix B. Estimates for Dirichlet series involving the PRincipal CHARACTER}

In this appendix we prove two estimates for Dirichlet series involving the principal character, and a bound for an oscillating integral, which were needed in $§ \S 2.3-2.5$. These results are of a rather standard type (see e.g. Lemma 8 of Hildebrand and Tenenbaum [5]), but we include the short proofs in the interests of completeness.

We suppose that $3 / 4 \leq \beta \leq 1$, say: in $\S \S 2.3-2.5$ we had $\beta=\alpha(x, y)$ or $\beta=$ $\alpha(x, y)-B k / \log x$. We also suppose that $\log q \leq \log ^{2} y$, which certainly implies that $\sum_{p \geq \log y, p \mid q} 1 / p \ll 1$. By partial summation from the prime number theorem, if $2 \leq z \leq$ $y$ and if $t \neq 0$ then

$$
\sum_{z \leq p \leq y} \frac{1-\cos (t \log p)}{p}=\log \log y-\log \log z-\int_{t \log z}^{t \log y} \frac{\cos w}{w} d w+O\left((1+|t|) e^{-d \sqrt{\log z}}\right),
$$

for a certain constant $d>0$. Choosing $z=e^{(\log \log y)^{3}}$, we find that if $1 / \log y \leq|t| \leq$ $1 /(\log \log y)^{3}$ then the sum is $\log \log y+\log |t|+O(1)$, whilst if $1 /(\log \log y)^{3} \leq|t| \leq$ $\log ^{1 / 4} y$, say, then the sum is $\log \log y-3 \log \log \log y+O(1)$. Thus we have

$$
\begin{aligned}
\left|\frac{L\left(\beta+i t, \chi_{0} ; y\right)}{L\left(\beta, \chi_{0} ; y\right)}\right| \leq e^{-\sum_{p \leq y, p \nmid q}(1-\cos (t \log p)) / p} & \ll e^{-\sum_{\log y \leq p \leq y}(1-\cos (t \log p)) / p} \\
& \ll \frac{\max \left\{|t|^{-1},(\log \log y)^{3}\right\}}{\log y}
\end{aligned}
$$

provided that $1 / \log y \leq|t| \leq \log ^{1 / 4} y$, and so

$$
\int_{\beta-i \log ^{1 / 4} y}^{\beta+i \log ^{1 / 4} y}\left|L\left(s, \chi_{0} ; y\right)\right|^{2} d|s|=O\left(\left|L\left(\beta, \chi_{0} ; y\right)\right|^{2} / \log y\right) .
$$

Next, we note that

$$
\left|\frac{L^{\prime}(\beta+i t, 1 ; y)}{L(\beta+i t, 1 ; y)}\right|=\left|\sum_{p \leq y} \frac{\log p}{p^{\beta+i t}-1}\right|=\left|\sum_{n \leq y} \frac{\Lambda(n)}{n^{\beta+i t}}\right|+O(1),
$$

since $\beta$ is large. By partial summation from the prime number theorem, if $y \geq 2$ then $\sum_{n \leq y} \frac{\Lambda(n)}{n^{\beta+i t}}=\frac{y^{1-\beta-i t}-1}{1-\beta-i t}+O\left((1+|t|) \int_{1}^{y} \frac{e^{-d \sqrt{\log w}}}{w^{\beta}} d w\right)=\frac{y^{1-\beta-i t}-1}{1-\beta-i t}+O\left((1+|t|) y^{1-\beta}\right)$, so if $\beta \leq \alpha=\alpha(x, y)$ then certainly

$$
\sum_{n \leq y} \frac{\Lambda(n)}{n^{\beta+i t}} \ll y^{\alpha-\beta} u \min \left\{\frac{\log u}{|t|}, \log y\right\}+(1+|t|) y^{\alpha-\beta} u \log u,
$$


on recalling the definition of $\alpha$. It follows, provided $\log u \leq \log ^{1 / 4} y$ (which certainly holds if $y$ is "large", in the sense of the introduction), that

$$
\int_{\beta-i \log ^{1 / 4} y}^{\beta+i \log ^{1 / 4} y}\left|\frac{L^{\prime}(s, 1 ; y)}{L(s, 1 ; y)}\right|^{2} d|s|=O\left(y^{2(\alpha-\beta)} u^{2} \log u \log y\right) .
$$

Finally note that if measurable $0 \leq \Phi \leq 1$ is supported on $[0,2]$, then

$$
\int_{t}^{\log ^{1 / 4} y} x^{i r} \breve{\Phi}(\beta+i r) d r=\int_{0}^{2} \Phi(v) v^{\beta-1} \int_{t}^{\log ^{1 / 4} y}(x v)^{i r} d r d v
$$

by definition of the Mellin transform. Splitting the integral over $v$ at $1 / \sqrt{x}$, and evaluating the integral over $r$, we find that this is $O(1 / \log x)$.

Acknowledgements. The author would like to thank Andrew Granville, Ben Green and Kannan Soundararajan for their encouragement, and for comments on a draft of this paper.

\section{REFERENCES}

[1] H. Davenport. Multiplicative Number Theory. Third edition, revised by H. L. Montgomery, published by Springer. 2000.

[2] R. de la Bretèche, G. Tenenbaum. Propriétés statistiques des entiers friables. The Ramanujan Journal, 9, pp 139-202. 2005

[3] A. Granville. Integers, without large prime factors, in arithmetic progressions. II Phil. Trans. R. Soc. Lond. A, 345, pp 349-362. 1993

[4] D. R. Heath-Brown. Zero-free regions for Dirichlet $L$-functions, and the least prime in an arithmetic progression. Proc. London Math. Soc., 64, pp 265-338. 1992

[5] A. Hildebrand, G. Tenenbaum. On integers free of large prime factors. Trans. Amer. Math. Soc., 296, no. 1, pp 265-290. 1986

[6] H. L. Montgomery. Ten Lectures on the Interface Between Analytic Number Theory and Harmonic Analysis. Published for the Conference Board of the Mathematical Sciences by the American Mathematical Society. 1994

[7] H. L. Montgomery, R. C. Vaughan. Multiplicative Number Theory I: Classical Theory. First edition, published by Cambridge University Press. 2007

[8] K. Soundararajan. The distribution of smooth numbers in arithmetic progressions. Anatomy of Integers, CRM Proc. and Lect. Notes, vol. 46, Amer. Math. Soc., Providence, RI, pp 115-128. 2008

[9] G. Tenenbaum. Introduction to analytic and probabilistic number theory. English edition, published by Cambridge University Press. 1995

Department of Pure Mathematics and Mathematical Statistics, Wilberforce Road, CAmbridge CB3 0WA, England

E-mail address: A.J.Harper@dpmms.cam.ac.uk 